\title{
Inspection Allocation Optimization with Resource Constraints Based on Modified NSGA-II in Flexible Manufacturing Systems
}

\author{
Yingchao You $\mathbb{D}^{1},{ }^{1}$ Guijiang Duan $\mathbb{D}^{1},{ }^{1}$ Rui Liu $\mathbb{D}^{1},{ }^{1}$ Taotao Liu $\mathbb{D}^{1},{ }^{1}$ and Mingcong Huang $\mathbb{D}^{2}$ \\ ${ }^{1}$ School of Mechanical Engineering and Automation, Beihang University, Beijing 100191, China \\ ${ }^{2}$ AVIC Chengdu Aircraft Industrial (Group) Co., Ltd, Chengdu 610091, China \\ Correspondence should be addressed to Guijiang Duan; gjduan@buaa.edu.cn
}

Received 2 June 2020; Revised 12 May 2021; Accepted 5 June 2021; Published 19 June 2021

Academic Editor: Bekir Sahin

Copyright ( 2021 Yingchao You et al. This is an open access article distributed under the Creative Commons Attribution License, which permits unrestricted use, distribution, and reproduction in any medium, provided the original work is properly cited.

With the development of smart manufacturing, quality has become an indispensable issue in the manufacturing process. Although there is increasing publication about inspection allocation problems, inspection allocation optimization research considering resource capability is scarce. This paper focuses on the inspection allocation problem with resource constraints in the flexible manufacturing system. Combined with the inspection resource capability model, a bi-objective model is developed to minimize the cost and balance loads of the inspection station. A modified NSGA-II algorithm with adaptive mutation operators is suggested to deal with the proposed model. Finally, a simulation experiment is conducted to test the performance of the modified algorithm and the results demonstrate that modified NSGA-II can obtain acceptable inspection solutions.

\section{Introduction}

In manufacturing systems, the quality characteristics (QC) of the manufactured product may inevitably fail to meet the requirement of the design because of machines, human error, etc. Therefore, inspection is a necessary part of the product manufacturing process [1]. Inspection is an organized assessment process, and it can be arranged after the processing procedure or even after the completion of the product. The quality and the condition of determining material of products will be inspected to know whether the product is suitable for use and the product meets the regulated requirement or not [2]. In reality, if there are too many rounds of inspection in the process of product manufacturing, it will raise the inspection cost and prolong the production cycle. Thus, many scholars conducted inspection process planning and inspection allocation research to arrange the inspection flow properly and dispatch the inspection resource, thus optimizing the cost of product inspection $[3,4]$.

Lindsay and Bishop [5] firstly studied the inspection allocation (IA) problem and proposed a perfect inspection model with the lowest cost for serial production. A dynamic planning model was used to get inspection solutions. Hurst et al. [6] built an imperfect inspection model which, for the first time, added the first and the second type of inspection errors into the inspection allocation model. Shiau et al. [7] built a model which integrated processing planning and inspection planning problems under limited resource. The model took a series of manufacturing systematic factors like manufacturing capability, inspection capability, and the cost of product quality into consideration to minimize the total cost. Korytkowski [8] proposed a multiproduct product inspection model, in which the inspection station was regarded as a $\mathrm{G} / \mathrm{G} / \mathrm{C}$ queuing model. The genetic algorithm was adapted to deal with the model to shorten the waiting time of inspection. Mohammadi et al. [1] brought up an optimizing framework for the inspection process and the framework aimed at two types of inspection, conformity inspection and monitoring inspection, balancing the cost and the quality.

Even many scholars researched the inspection allocation problem. However, the problems that current research mainly focused on are the inspection location and inspection process planning. Many scholars regarded the inspection stations as the same inspection unit, which is an ideal processing way to some extent. In the actual condition, 
different inspection stations own specific inspection capabilities, such as precision, process time. Therefore, in this paper, two subproblems are taken into consideration. The first one is the inspection location problem and the second one is the inspection resource allocation problem based on the inspection capability model of Shiau [9]. Following the inspection process planning definition of Narahari et al. [10], the paper summarized the inspection allocation problem with resource constraints as follows:

(i) The location of inspection in the product manufacturing cycle.

(ii) Which inspection station will be used to inspect the QC?

For the type of manufacturing system, people paid more attention to the research on the IA problem in the series manufacturing system. There are few papers concerning the IA problem in the Flexible Manufacturing System (FMS). In the FMS, the product flow and inspection flow of a production line have many types of parts. The inspection stations shall inspect different types of components that contain separate product QCs, and each of the components owns a specific inspection plan. Therefore, the allocation of inspection resource becomes very complicated in a complete view.

On the other hand, plenty of scholars took the inspection cost, quality improvement, and uncertain factors into consideration in the inspection allocation problem. The inspection unit is a crucial resource, and its working load is worthy of concern. However, it seldom was mentioned by scholars. When the inspection station is put into use and each working unit gets a relatively balanced working quantity, the overall inspection efficiency will be improved. Therefore, in the paper, a multi-objective inspection allocation with resource constraints (IARC) model is built in the FMS to trade-off manufacturing cost and inspection station load. This research looks forward to reducing the planning difficulty in the inspection allocation and giving support to decision-making.

The methodology to solve the inspection planning problem varies, such as dynamic programming, integrated programming [11]. With the increase of system size, the amount of computation of previous methods increased and these methods became impractical [12]. In recent years, more and more scholars have paid attention to heuristic algorithms and metaheuristic algorithms to solve the inspection planning problem, which belongs to an NP-hard problem [13]. Especially, genetic algorithm (GA) is the most suitable method to deal with the inspection allocation problem as the codes express the inspection policy directly. Hsin Rau et al. [12] proposed a GA approach for solving the inspection allocation problem, and they also analysed the similarity of the codes used in the chromosome of the GA approach and the representation of the inspection allocation policy for workstations in the production system. Due to the complexity of inspection planning problems, some scholars treated them as multi-objective optimization problems and proposed corresponding solutions.
Mohammadi et al. [14] presented a bi-objective, mixed-integer linear programming (BOMILP) model to tackle the inherent uncertainty of the concerned parameters. A differential evolution algorithm combined with the Taguchi and Monte Carlo methods was proposed to solve the model. Besides, simulation methods were also used in this problem [15]. However, simulation just investigated a limited number of production scenarios for optimization.

In this paper, the proposed IARC model belongs to a multi-object optimization problem (MOP), in which objects contract with each other. Thus, finding the optimal solutions for every object is impossible. Multi-objective heuristic algorithms are practical to solve the MOP problem; many methods were developed in recent years, such as NSGA-II, NASG-III, MOEA/D, Monarch butterfly optimization, and differential evolution algorithm, quantum-inspired differential evolution [16-20]. For the inspection allocation problem, the methods for a single-objective problem are abundant, such as the dynamic programming algorithm, genetic algorithm [12, 21-24]. For multi-objective problems, there are a fewer number of methods than methods in the single-objective problem. NSGA-II is one of the most popular methods, and it is used to solve the inspection planning problem and obtain an acceptable result [11]. In this paper, the modified NSGA-II algorithm is used for solving the IARC model.

The following are the main contributions of this paper:

(1) Inspection-capability-based IARC is the first model which allocates inspection location and inspection station in the FMS. Besides, the IARC is a bi-objective optimization model which tries to trade-off the cost and machine load.

(2) A modified algorithm is proposed with three adaptive mutation operators for the IARC model. The simulation experiment validates the performance of the proposed algorithm.

The remainder of the paper is organized as follows. In the next Section, problem description and mathematical modeling are presented. In Section 3, a modified NSGA-II with three kinds of mutation operators is described. In Section 4, simulation and analysis are conducted to verify the effectiveness of the model and algorithm. Finally, conclusions are stated in Section 5.

\section{Problem Description and Mathematical Modeling}

2.1. Problem Description. The FMS production line includes many types of parts. Here, $\Omega=\{1, \ldots, P\}$ is used to express parts. A part $p \in \Omega$ has $K_{p}$ quality characteristics $\mathrm{QC}_{p, q}$, and each $\mathrm{QC}_{p, q}$ owns the target value $M_{p, q}$, upper deviation $\mathrm{USL}_{p, q}$, and the lower deviation $\mathrm{LSL}_{p, q}$. In the system, there are machine tools $M=\{1, \ldots, N\}$ and inspection stations $\Lambda=\{1, \ldots, I\}$. According to the production technique, the work in process (WIP) gets $\mathrm{QC}_{p, q}$ after being processed in the machine $n$. If the next procedure is not an inspection for 
part $p$, it will be transferred to the next working procedure. If the component should be inspected, then it will be conveyed to the inspection station $i \in \Lambda$ for inspection. The products in the system share the same manufacturing resource $M$ and inspection resources $\Lambda$. The inspection strategy for manufactured products is as follows: qualified (to the next procedure), reworked, and discarded. When the inspection value $\mathrm{LSL}_{p, q}<v_{p, q}<\mathrm{USL}_{p, q}$, the product is qualified and shall be put into the next procedure. When $v_{p, q}>\mathrm{USL}_{p, q}$, the product shall be reworked. When $v_{p, q}<\mathrm{LSL}_{p, q}$, the product is not qualified and will be discarded.

The workstation $n$ and inspection station $i$ have their specific resource capability. Therefore, the first and second types of errors exist. The actual value $x$ of processing $\mathrm{QC}_{p, q}$ of processing station $n$ is assumed to be subjected to the normal distribution $f(n) \sim N\left(u_{n}, \sigma_{n}\right)$. Similarly, the measured value $y$ of inspection $\mathrm{QC}_{p, q}$ of the inspection station is assumed to be subjected to the normal distribution $\varphi_{i} \sim N\left(u_{i}, \sigma_{i}\right)$. The inspection results after processing of QC could be divided into 7 types. For specific content, please refer to Table 1, and for the calculation regulation, please refer to Shiau [25].

Pursuing lower costs is a vital issue in manufacturing [25]. Costs in the production process include manufacturing cost, inspection cost, scrap cost, and loss cost of defective product sold to customers. Inspection plans have an indirect effect on costs as inspection will affect the products' flow in manufacturing. If the inspection procedure increases, the inspection cost will increase, but the subsequent manufacturing cost will be reduced as the defective goods will be detected out in advance in manufacturing [14]. At the same time, defective goods will be prevented from flowing into the market. The decrease in the number of defective goods flowing into the market will reduce the damage caused by defective goods. Therefore, the total cost is the first objective function obj1 of the IARC model. In the FMS, the inspection unit shall process many kinds of inspection tasks. The method of assigning tasks to inspection stations is worth studying. When each inspection station is assigned a relatively balanced inspection task, the overall inspection efficiency will be improved. To prevent the large task gap between inspection stations, the maximum load of the inspection unit is the second objective function $\mathrm{obj}_{2}$.

\subsubsection{Model Hypothesis}

(1) In manufacturing, each machine tool processes only one product $Q C_{p, q}$

(2) In an inspection procedure, each inspection station inspects only one $Q C_{p, q}$

(3) Suppose that the whole system is working correctly, and the processing flow is known

\subsubsection{Parameters of the IARC Model}

$C_{i, p, q}$ : the expected probability that conforming units are correctly classified as qualified units by inspection station class $i$
TABLE 1: The inspection probability based on the resource capability.

\begin{tabular}{lccc}
\hline$y_{x}$ & $x<L S L_{p, q}$ & $L S L_{p, q} \leq x \leq U S L_{p, q}$ & $x>U S L_{p, q}$ \\
\hline$y<L S L_{p, q}$ & $S l_{n, i, p, q}$ & $\alpha l_{n, i, p, q}$ & - \\
$L S L_{p, q} \leq y \leq U S L_{p, q}$ & $\beta l_{n, i, p, q}$ & $C_{n, i, p, q}$ & $\beta u_{n, i, p, q}$ \\
$y>U S L_{p, q}$ & - & $\alpha u_{n, i, p, q}$ & $S u_{n, i, p, q}$ \\
\hline
\end{tabular}

$S l_{i, p, q}$ : the expected probability that nonconforming units with quality characteristics $<\mathrm{LSL}_{p, q}$ are correctly classified as defective units by inspection station class $i$

$\mathrm{Su}_{n, i, p, q}$ : the expected probability that nonconforming units with quality characteristics $>\mathrm{USL}_{p, q}$ are correctly classified as defective units by the inspection station $i$ $\alpha l_{i, p, q}$ : expected Type I error that conforming units are incorrectly classified as defective units with quality characteristics $<\mathrm{LSL}_{p, q}$ by inspection station class $i$

$\alpha u_{n, i, p, q}$ : expected Type I error that conforming units are incorrectly classified as defective units with quality characteristics $>\mathrm{USL}_{p, q}$ by inspection station class $i$

$\beta l_{\mathrm{i}, \mathrm{p}, \mathrm{q}}$ : expected Type II error that nonconforming units with quality characteristics $<\mathrm{LSL}_{p, q}$ are incorrectly classified as qualified units by inspection station class $i$

$\beta u_{i, p, q}$ : expected Type II error that nonconforming units with quality characteristics $>\mathrm{USL}_{p, q}$ are incorrectly classified as qualified units by inspection station class $i$ $\mathrm{MC}_{p, q}$ : unit manufacturing cost of $\mathrm{QC}_{p, q}$ $\mathrm{ICi}_{p, q}$ : unit inspection cost of $\mathrm{QC}_{p, q}$ by using inspection $i$

$\mathrm{SC}_{p, q}:$ the scrap cost of product $p$

$\mathrm{RC}_{p, q}:$ the rework cost of product $p$ of the $\mathrm{QC}_{p, q}$

$\mathrm{CSC}_{p, q}$ : loss cost of defective product sold to customers due to the $\mathrm{QC}_{p, q}$

$T C$ : the total cost of all products

$T i$ : the total inspection time of inspection $i$

\subsubsection{Decision Variables}

$\omega_{p, q}:$ the probability of product $p$ can reach stage $q$ $h_{p, q}=1: \mathrm{QC}_{p, q}$ need an inspection according to the inspection plan, otherwise 0

$w_{p, q}, i=1: \mathrm{QC}_{p, q}$ need to be inspected by the inspection station $I$ according to the inspection plan, otherwise 0

\subsection{Problem Analysis}

2.2.1. Product/Inspection Flow. After inspecting $\mathrm{QC}_{p, q}$, the processing strategy includes rework, discard, and entering the next step. If the $\mathrm{QC}_{p, q}$ does not need an inspection, the product can enter the next procedure directly. While the $\mathrm{QC}_{p, q}$ needs an inspection, the qualified one can enter the next working procedure. Even the second type of error occurs, namely, the defective goods will also enter the next step by mistake. 
The probability of product $p$ in the $q$ working procedure could flow into the next working procedure:

$$
\eta_{p, q}=1-h_{p, q}+h_{p, q}\left(\beta l_{i, p, q}+C_{i, p, q}+\beta u_{i, p, q}\right) .
$$

The probability of product $p$ in the $q$ working procedure being discarded:

$$
\mu_{p, q}=h_{p, q}\left(S l_{i, p, q}+\alpha l_{i, p, q}\right) .
$$

The probability of product $p$ in the $q$ working procedure being reworked:

$$
v_{p, q}=1-\eta_{p, q}-\mu_{p, q} .
$$

The probability of the product $p$ entering the $q$ working procedure:

$$
\omega_{p, q}=\omega_{p, q}-1 \eta_{p, q-1}
$$

2.2.2. Cost Model. Following the possible results of the product after each working procedure, we can calculate the inspection cost, manufacturing cost, reworking cost, discarding cost, and damage cost caused by selling the defective goods to the customer. Based on the above probabilities, we can get the related costs as follows:

The manufacturing cost of product $p$ :

$$
T M C_{p}=\sum_{q=1}^{Q} M C_{p, q} \omega_{p, q} .
$$

The inspection cost of product $p$ :

$$
\operatorname{TIC}_{p}=\sum_{q=1}^{Q} h_{p, q} \omega_{p, q} I C_{i, p, q} .
$$

The reworking cost of product $p$ :

$$
T R C_{p}=\sum_{q=1}^{Q} v_{p, q} \omega_{p, q} R C_{p, q} .
$$

The damage caused by discarding the product $p$ :

$$
\operatorname{TSC}_{p}=\sum_{q=1}^{Q} v_{p, q} \mu_{p, q} S C_{p, q} .
$$

The damage caused by selling the defective goods $p$ to the customer:

$$
\operatorname{TCSC}_{p}=\sum_{q=1}^{Q} \operatorname{CSC}_{p, q} \omega_{p, q}\left(\beta l_{i, p, q}+\beta u_{i, p, q}\right) .
$$

By integrating all the cost factors, and we can get the total cost of product $p$.

$$
T C_{p}=T M C_{p}+\text { TIC }_{p}+\text { TRC }_{p}+\text { TSC }_{p}+\text { TCSC }_{p} .
$$

2.2.3. Time Model. Following the distribution condition of the inspection station, the total working time of inspection station $i$ is:

$$
T_{i}=\sum_{p=1}^{Q} \sum_{q=1}^{Q} t_{i, p, q} w_{p, q, i}
$$

2.3. Mathematic Model. Aiming at the optimization problem of inspection allocation under the FMS, the minimum total cost and minimum biggest inspection station load were taken as targets to build a multi-objective MIP model:

Objective function:

$$
\begin{aligned}
& \mathrm{obj}_{1}: \min (C), \\
& \mathrm{obj}_{2}: \min \left(T_{\max }\right), \\
& T_{\text {max }} \geq T_{i}, \forall i \in \wedge, \\
& C \geq \sum_{p=1}^{P}\left(T M C_{p}+T I C_{p}+T R C_{p}+T_{\left.S C_{p}+T C S C_{p}\right),}\right. \\
& h_{p, q}=\sum_{i=1}^{I} W_{p, q, i}, \quad \forall p \in \Omega, \forall q=1 \ldots K_{p}, \\
& W_{p, q, i} h_{p, q}=1 \text { or } 0, \quad \forall p \in \Omega, \forall q=1 \ldots K_{p}, \forall i \in \wedge, \\
& T M C_{p}, T I C_{p}, T R C_{p}, T S C_{p}, T C S C_{p}, t_{i, p, q}>0, \quad \forall p \in \Omega, \forall q=1 \ldots K_{p}, \forall i \in \wedge .
\end{aligned}
$$

Formula (12) expresses how to minimize the total cost of all the products. Formula (13) expresses how to minimize the maximum inspection time among all inspection stations. Formula (14) shows the relation between the maximum load 
and the working time of inspection stations. Formula (15) represents the relation between the total cost and related costs. Formula (16) expresses that each QC shall be arranged to one inspection station. In formula (17), $w_{p, q, i}, h_{p, q}$ are the variates of $0-1$. Formula (18) means that the value of costs and time are positive numbers.

\section{The Modified NSGA-II Algorithm}

The IARC model mentioned in this paper includes two fitness function formulas, (12) and (13). To address this model, NSGA-II is adopted to calculate the Pareto optimal solution of targets and balance these two optimizing targets. Besides, some necessary modification is done with the algorithm to adapt the IARC model and enhance the performance. A four-layer coding scheme for chromosomes is put forward, and it can express the inspection locations and inspection resource assignment scheme directly. In the mutation scheme, three types of mutation operators are raised to enhance neighborhood searching capability. The multipoint crossover method is adopted to the NSGA-II algorithm. The flow chart of the modified NSGA-II is shown in Figure 1. The crowding distance calculation and nondominated sorting method are adopted in this paper referring to Deb [26].

3.1. Coding. The coding scheme adopted four-layer chromosomes to express the inspection location and inspection resource assignment scheme, and it is shown in Figure 2. The first layer is the production layer, and the value of each unit in the layer is the serial number of the products. The second layer is the QC layer, which shows the serial number of QCs. The third layer shows the inspection scheme. When the QC needs inspection according to the plan, the value is 1 , otherwise it is 0 . The fourth layer is the resource assignment layer, which shows the serial number of inspection stations. When the value is 0 , the associated QC does not need an inspection. Each layer of chromosomes is a task unit $\mathrm{O}$, which contains information on inspection strategy and inspection resource. For instance: $\mathrm{O}(1,1,1,1)$ means that the $\mathrm{QC}_{1,1}$ shall be inspected in inspection station 1 after processing. It is worth noting that layer one and layer two are static in the evolution process.

3.2. Population Initialization. This paper proposed three ways for initial solution generation: (1) 20\% Random Generation Method (RGM), (2) 40\% Minimum Load Selection Method (MLSM), and (3) 40\% Earliest Stage Assignment Method (ESAM). The ESAM was proposed by Shiau [25]. In ESAM, the earlier the manufacturing procedure is, the higher weight the QC will be allocated. There is a higher probability that this QC will be allocated an inspection station. The MLSM selects the inspection station with the minimum weight among all the available resources to inspect the QC. The detailed steps of the initial solution generation are as follows:
Step 1: All QCs are traversed in order. When the QC exists, skip to step 2. When all the QCs have been traversed, end.

Step 2: The 0-1 variable $v_{1}$ is generated randomly. When $v_{1}=1$, the QC needs an inspection, and then skip to step 3 to allocate the inspection station. When $v_{1}=0$, the $\mathrm{QC}$ does not need an inspection and returns to step 1.

Step 3: A random number $v_{2}$ between [0-1] is generated. When $v_{2} \leq 0.2, \quad \mathrm{RGM}$ is selected. When $0.2<v_{2} \leq 0.6$, MLSM is selected. When $0.6<v_{2} \leq 1$, ESAM is selected. Finally, skip back to Step 1 .

3.3. Multipoint Crossover Method. The multipoint crossover method is adopted in this algorithm. $P 1$ and $p 2$ are paternal chromosomes, and $c 1$ and $c 2$ are, respectively, the offspring chromosomes generating from $p 1$ and $p 2$. Two cross positions are selected randomly and gene fragments of $p 1$ and $p 2$ are exchanged. Finally, the new generations $c 1$ and $c 2$ are created. The crossover method is shown in Figure 3.

3.4. Adaptive Mutation Operators. The most common mutation method is single point variation, which has a shortage. The generated population has a low diversity, and it further makes the solutions fall into the local optimal solution prematurely. Besides, multilayer encoding is characteristic of deep dependency between different layers of genes. If the method of single point variation is adopted, the infeasible offspring may be created, as the other layer of genes may not change with the mutated gene.

In recent years, neighborhood search strategy has been successfully applied to combinatorial problems with good performance [27]. In this section, three different variation strategies are proposed by combining the neighborhood search strategy with variation strategy to improve the diversity of the population. The mutation operator selects the task to be optimized based on the current population. Once the task subset is selected, the corresponding variable is deemed to be optimized. Mutation can be considered as a neighborhood search operation on an existing solution structure. The specific contents include:

(1) Random inspection task change operator (RIC) : RIC randomly selects a $\mathrm{QC}$ and changes the inspection policy of the QC. If QC does not need inspection before, this QC is reassigned as an inspection procedure. Then, an available inspection station will be allocated to the QC. If the QC needs an inspection, the inspection policy of the QC is cancelled.

(2) Random equipment replacement operator (RER): This operator can randomly select an inspection procedure and randomly reassign a piece of available equipment for this inspection procedure.

(3) Maximum load removal operator (MLR) : A total load of all devices is calculated and the inspection station $i$ with the maximum load is selected. The chromosome fitness value effectively increases if 


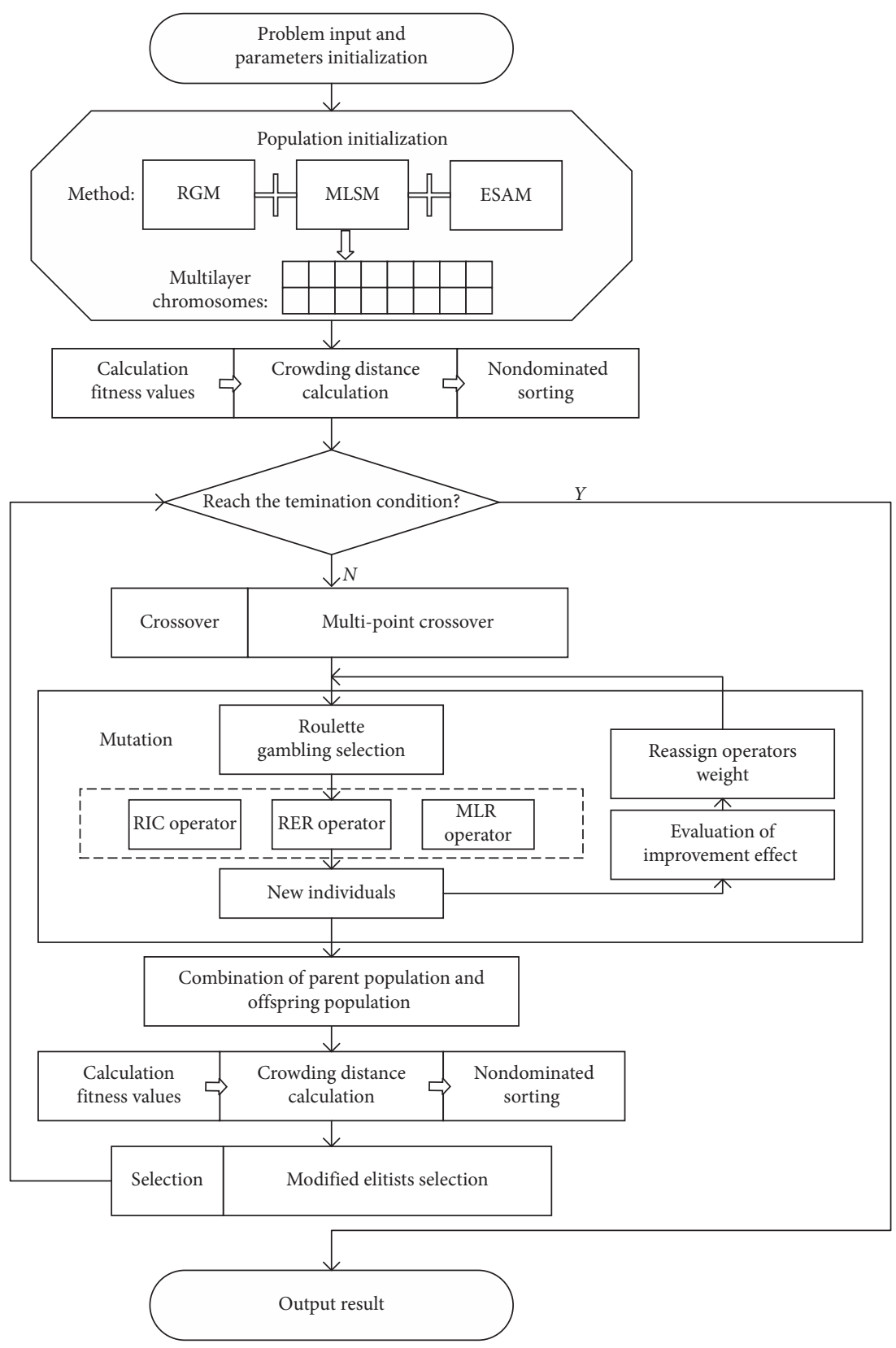

FIGURE 1: Flow chart of Modified NSGA-II algorithm.

\begin{tabular}{c|c|c|c|c|c|c|c|c|c|c|c|c|}
\hline Product layer & 1 & 1 & 1 & 1 & 2 & 2 & 2 & 2 & 2 & 3 & 3 & 3 \\
\cline { 2 - 11 } QC layer & 1 & 2 & 3 & 4 & 1 & 2 & 3 & 4 & 5 & 1 & 2 & 3 \\
\cline { 2 - 11 } Location layer & 1 & 0 & 1 & 0 & 1 & 0 & 0 & 1 & 1 & 0 & 1 & 1 \\
\cline { 2 - 11 } RS layer & 1 & 0 & 3 & 0 & 4 & 0 & 0 & 2 & 5 & 0 & 2 & 3 \\
\cline { 2 - 10 } & & & &
\end{tabular}

Figure 2: Coding scheme of modified NSGA-II.

the bottleneck factors are improved. A random inspection task which should be inspected by the inspection station $i$ will reallocate another inspection station.
The mutation operators are designed based on human experience on the inspection allocation problem. The three mutation strategies differ from each other but do not have the same importance. In the process of population evolution, 


\begin{tabular}{c}
$p 1$\begin{tabular}{|l|l|l|l|l|l|l|l|l|l|l|l|}
\hline 1 & 1 & 1 & 1 & 2 & 2 & 2 & 2 & 2 & 3 & 3 & 3 \\
\hline 1 & 2 & 3 & 4 & 1 & 2 & 3 & 4 & 5 & 1 & 2 & 3 \\
\hline 1 & 0 & 1 & 0 & 1 & 0 & 0 & 1 & 1 & 0 & 1 & 1 \\
\hline 1 & 0 & 3 & 0 & 4 & 0 & 0 & 2 & 5 & 0 & 2 & 3 \\
\hline$c 1$ \\
\hline 1 & 2 & 3 & 4 & 1 & 2 & 3 & 4 & 5 & 1 & 2 & 3 \\
\hline 1 & 0 & 1 & 1 & 0 & 1 & 1 & 1 & 1 & 0 & 1 & 1 \\
\hline 1 & 0 & 3 & 5 & 0 & 3 & 1 & 2 & 5 & 0 & 2 & 3 \\
\hline
\end{tabular} \\
\hline
\end{tabular}

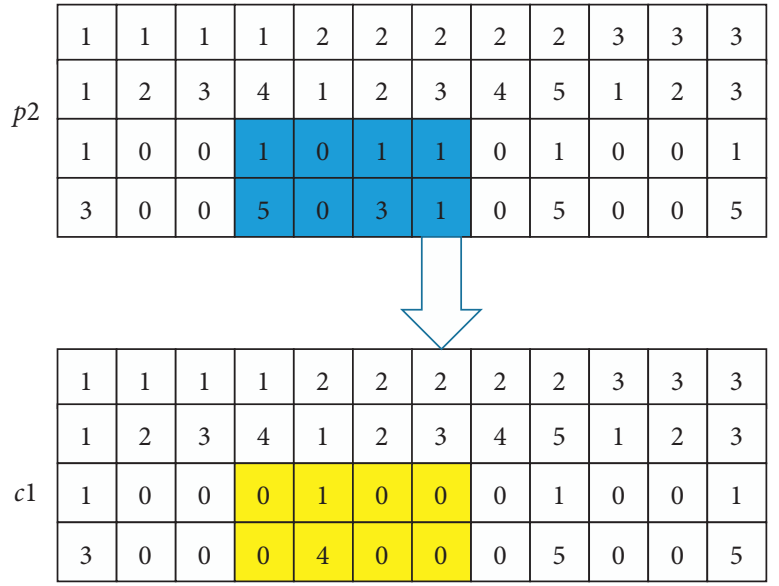

FIGURE 3: Multipoint crossover method.

an adaptive selection strategy is proposed. For each generation of chromosome mutation, roulette is used to select the mutation operator according to the weight of the mutation operator $w_{\theta}, \theta \in \Theta$. The initial value $w_{\theta}$ is 1 . With the increase of generation, the mutation operator weights $w_{\theta}$ are updated according to the improvement of chromosome fitness $S_{\text {gen }}$ after mutation.

The computation rules are as follows:

$$
\begin{aligned}
w_{\theta} & =w_{\theta}+S_{\text {gen }}, \\
S_{\text {gen }} & =\frac{o b j 1_{\text {gen }}-\text { obj } 1_{\text {gen-1 }}}{\text { obj } 1_{\text {gen }-1}}+\frac{o b j 2_{\text {gen }}-o b j 2_{\text {gen }-1}}{\text { obj } 2_{\text {gen }-1}} .
\end{aligned}
$$

3.5. Modified Elite Selection Strategy. Selection is an essential operation, which transmits excellent and adaptable parents to the next generation and maintains the quantity of the population unchanged. The individuals in the population are divided into different frontiers of inferior quality according to govern relations by using the nondominated sorting method. The elite selection strategy always retains the nondominant solution in the lower frontier to the next generation. In this case, the majority of chromosomes come from level one of the nondominant frontier of the last generation. Thus, the optimal result always entraps into the local optimum solution. The elite selection strategy is improved by adjusting the probability of the lower-level solution to the next generation. The specific steps are as follows:

Step 1: The chromosomes of the parent and offspring are combined $P_{a i}=C h_{i} \cup F_{a i}$. The total quantity of chromosomes is $\left|F_{a i}\right|$. The individuals are divided into several frontiers, which are expressed as $F_{t}=\left\{F_{1}, \ldots\right.$, $\left.F_{i}\right\}$. The number of optimal solutions at inferior $t$ is $\left|F_{t}\right|$.

Step 2: The next generation is $F_{a i}+1=\varnothing$, and the initial Pareto frontier is $t=1$.

Step 3: The quantity of individuals that are selected to the next generation at inferior $t$ is $n_{i}=(1-0.1 t)\left|F_{t}\right|$. If
$n_{i}+\left|F_{a i}+1\right| \leq\left|\mathrm{Ch}_{i}\right|$, execute step 4. Otherwise, execute step 5 .

Step 4: The individual at the present level $\left|F_{t}\right|$ and the parent generation $P_{a i}+1$ are combined, $P_{a i}=\mathrm{Ch}_{i} \cup F_{a i}+1 t=t+1$. Skip to the last step.

Step 5: Selection finished.

\section{Simulation and Analysis}

In this Section, a simulation experiment is conducted to verify the correctness of the proposed inspection planning model and the feasibility of the modified NSGA-II algorithm. The experiment bases on a flexible production line of an aircraft manufacturer and the parameters are estimated following Shiau [25]. The proposed inspection planning model belongs to a multi-objective programming problem whose outcomes are normally difficult to evaluate. Thus, a quantified indicator called "hyper-volume indicator" is adapted to evaluate the performance of NSGA-II, modified NSGA-II. Besides, the outcome of a specific example is listed individually to analyze the inspection solutions and related cost.

4.1. Parameters Setting. Based on the operational situation of a factory, 5 machine tools and 3 inspection tools were set in the experiment. Process capability $\sigma_{n}$ of machine tools and inspection capability $\sigma_{i}$ of the inspection stations are normally distributed, and the specific parameters of the normal distribution are shown in Table 2. There are two classes of parts, class $A$ and class $B$. Class $A$ has a relatively simple structure, and 4-5 QCs are in this category. Another category class B has 8-10 QCs, and all QCs can be machined or inspected by the tools mentioned above. The $L S L, U S L$, and the related costs of QC are shown in Table 2.

The experiment has 17 inspection planning tasks that deal with different types of parts. For example, the instance I6_A has 6 kinds of parts, which are randomly selected from class $A$. The instance I6_AB means there are 6 kinds of parts. The half parts are randomly selected from class $A$, and the 
TABle 2: Parameters of the simulation experiment.

\begin{tabular}{lc}
\hline Parameter & Range \\
\hline$\sigma_{n}$ & $0.1-0.6$ \\
$\sigma_{i}$ & $0.001-0.06$ \\
{$[L S L-U S L]$} & $2-3 \sigma_{n}$ \\
$M C$ & $20-80$ \\
$I C$ & $10-20$ \\
$S C$ & $800-2000$ \\
$R C$ & $20-80$ \\
$C S C$ & $200-500$ \\
\hline
\end{tabular}

others are from class $B$. All the instances are shown in Table 3.

To verify the effectiveness of the proposed algorithm, NSGA-II and modified NSGA-II are used to calculate the examples. Matlab R2020a was used to implement the program, which was run on the Intel $(R)$ Core (TM) i5-7200 $2.50 \mathrm{GHz}$ computer under the Windows 7 operating system. The parameters of both algorithms were set as follows: popsize $=50, \quad$ max-_gen $=100, p_{c}=0.75$. The mutation probability for NSGA-II is only 0.25 . To evaluate the performance of the two algorithms, the following evaluations of the algorithm are adopted.

4.2. Metric and Objective Normalization. The hyper-volume $(\mathrm{HV})[28,29]$ is a qualitative metric that can evaluate the convergence and diversity of multi-objective problems synchronously $[30,31]$. This metric is used by calculating the volume of the objective space that is covered by individuals of a nondominated solution set. The value of $\mathrm{HV}$ depends on the number of objective functions, the number of individuals in the nondominated solutions set, and reference points. The equation is

$$
H V(N D, A)=\wedge\left(U h\left(D_{l}\right) \mid D_{l} \in N D, \quad l=1, \ldots, \gamma\right),
$$

where ND is a nondominated solution set $\left\{D_{1}, D_{2}, \ldots, D\right.$ Ү,$\quad h\left(D_{l}\right)=\left|D_{l 1}-A_{1}\right| \times\left|D_{l 2}-A_{2}\right| \times \ldots \times\left|D_{l c}-A_{c}\right| ; c$ is the number of objective functions.

Normalization of the objective function values is required before calculating the hyper-volume to obtain a nondimensionalised optimization problem [32]. A simple normalization method is adapted to replace the objective [33]. Through normalization, the values of objective functions range from $[0,1]$.

$$
\bar{f}^{i}=\frac{f_{i}-z_{i}^{*}}{z_{i}^{\text {nad }}-z_{i}^{*}},
$$

where $z_{i}^{*}$ is the max point in the objective space, $z_{i}^{\text {nad }}$ is the nadir point in the objective space.

4.3. Result Analysis. This section consists of two parts: the outcome analysis and performance analysis. The outcome analysis aims to analyse the costs and the load of inspection tasks by comparing the result of a specific inspection task leveraging by the IARC model and the NSGA-II algorithm.
TABLE 3: The instances of the experiment.

\begin{tabular}{lllllllll}
\hline I1_A & I1_B & NA & I2_A & I2_B & I2_AB & I4_A & I4_B & I4_AB \\
\hline
\end{tabular}

The objective of performance analysis is to compare the performance of NSGA-II and modified NSGA-II in the inspection planning problem by using the $\mathrm{HV}$ metric.

4.3.1. Outcome Analysis. The inspection instance I4_A was selected as an example to carry out the outcome analysis. Three methods, namely, Modified NSGA-II, NSGA-II, and all inspection strategy, were adapted to deal with this inspection task. All inspection strategy means that all the QCs of 4 parts from class $A$ will be inspected by 3 inspection stations, and the inspection stations were allocated to these inspection tasks randomly. Finally, the result is obtained, and the selected scheme, the costs, the objective value, and the HV are shown in Table 4.

The obj1 value is the sum of MC, IC, DC, RC, and CNC, and it means that all factors affect the result collectively. It is clear that the proportion of $\mathrm{MC}, \mathrm{DC}$, and $\mathrm{CNC}$ is relatively large among all factors by three methods, but the value of MC changes slightly among the three plans. Thus, it has a slight influence on obj1 as the manufacturing operations are inevitable. For the comparison of the outcome of NSGA-II and modified NSGA-II, there are more nondominated solutions by using modified NSGA-II. By comparing the outcome of all inspection strategies with heuristic algorithms, the results from the optimization algorithm are distinctly superior to the solutions from all inspection strategies. Especially, the cost of inspection and discard is visibly higher than the other two methods, because more inspection operations were set in the plan and more defective products were taken out along the process. The value of CNC in all inspection strategy is lower than others, as the inspection is strict and defective products can seldom flow into the market.

For the comparison of the two optimal algorithms, please refer to Figure 4, which shows the distribution of nondominated solutions in the objective space of two algorithms. The horizontal axis represents the total cost, and the vertical axis represents the load balance. The modified NSGA-II can generate more nondominated solutions compared with NSGA-II, and the solutions of modified NSGA-II are dominated to the nonmodified one. To further compare the performance of the two algorithms, performance analysis is done in the next section.

4.3.2. Performance Analysis. Performance analysis is conducted by dealing with the 17 instances provided in Table 3 ten times using two algorithms, NSGA-II and the modified NSGA-II. The HV is used to assess the results, and statistical analysis is carried out according to the results. The statistic variables are shown in Table 5.

For the mean value of 10 times runs, both the Modified NSGA-II and NSGA-II can see a slight growth in mean value 
TABLE 4: The result of I4_A by using three methods.

\begin{tabular}{|c|c|c|c|c|c|c|c|}
\hline Methods & $N$ & & $\begin{array}{c}\text { Selected Scheme } \\
\text { P1/P2/P3/P4 }\end{array}$ & $\begin{array}{c}\text { Costs } \\
\mathrm{MC} / \mathrm{IC} / \mathrm{DC} / \mathrm{RC} / \mathrm{CNC}\end{array}$ & $\mathrm{obj}_{1}$ & $\mathrm{obj}_{2}$ & $\mathrm{HV}$ \\
\hline Modified NSGA-II & 8 & $\begin{array}{l}\text { LI } \\
\text { IA }\end{array}$ & $\begin{array}{c}00011 / 00101 / 00100 / 01000 \\
00031 / 00103 / 00200 / 01000\end{array}$ & $569 / 31 / 418 / 41 / 743$ & 1800.7 & 65 & 0.89 \\
\hline NSGA-II & 2 & $\begin{array}{l}\text { LI } \\
\text { IA }\end{array}$ & $\begin{array}{l}00011 / 00101 / 10100 / 01000 \\
00011 / 00103 / 10200 / 02000\end{array}$ & $561 / 52 / 418 / 41 / 733 /$ & 1805.8 & 74 & 0.85 \\
\hline All inspection & - & $\begin{array}{l}\text { LI } \\
\text { IA }\end{array}$ & $\begin{array}{l}11111 / 11111 / 11111 / 11111 \\
31333 / 22231 / 13333 / 22212\end{array}$ & $380 / 351 / 992 / 30 / 358$ & 2111.5 & 4536 & - \\
\hline
\end{tabular}

Note. $\mathrm{LI}=$ location of inspection station; RA = inspection station assignment scheme. $N$ = the number of solutions in nondominated solutions set; “-" means not available.

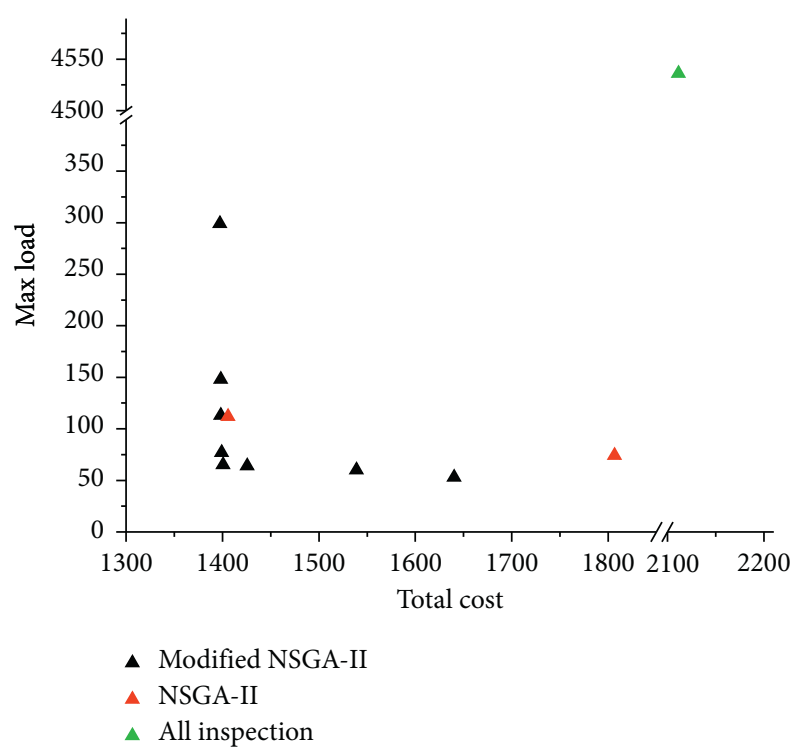

Figure 4: Comparison of Pareto front of three methods.

as the number of products involved in the experiment increased. When there is only one product in the experiment, the difference between the mean values of the two algorithms is tiny. The gap between the two results becomes large when the number of products involved in the experiment increased. The largest difference between the two algorithms is $7.73 \%$ in $I 10 \_A B$ and the average difference of the mean value of all instances in 10 runs is $3.36 \%$. It means that the modified NSGA-II is excellent when dealing with complex inspection allocation task and the modified NSGA-II outperforms NSGA-II in dealing with the IARC model. The variance of the modified NSGA-II is smaller than the corresponding value of NSGA-II in most cases, which means that the solutions of the previous algorithm converge evidently than the latter one. The average number of solutions in the nondominated solutions set generated by modified NSGA-II is far more than the other one and it means that the previous one performs well in terms of variety. For the performance of algorithms in different tasks, the modified NSGA-II performs better when dealing with hybrid class AB than class A and class B. Figure 5 shows a box plot of the HV metric in I4_A.

The computation complexity of the algorithms is compared, and the average time of ten runs is listed in
Table 5. The average runtime of modified NSGA-II is higher than NSGA-II by around $8 \%$. The reason is that the adaptive mutation operators need more extra time, which is in an acceptable range. Figure 6 shows a box plot of runtime in example I4_A, and there is no significant difference between the two algorithms in terms of runtime.

It is worth mentioning that the performance of different mutation operators is different when dealing with the IARC model. The performance is evaluated by using the average changes of weight value for mutation operators, which is shown in Table 6. The initial values of all operators are the same. Thus, the larger the value of average changes, the greater the contribution of the method to objective optimization. The MLR is the best mutation operator in this experiment. RIC is the second one, and the RER has the worst performance. The MLR focuses on the load balance to optimize the obj ${ }_{2}$. In the simulation, the value of $\mathrm{obj}_{2}$ improvement is large, which can be concluded by observing the $\mathrm{obj}_{2}$ in Table 4. Therefore, the MLR has the best performance in this experiment.

\section{Discussion}

Through the outcome analysis, the wrong idea that all inspection strategy is always the most economically beneficial solution was corrected, but all inspection strategy is widely adopted in some factories. By using the quantitative methods, the lowest-cost solution could be found. However, the formulation of the method often considers more than a single factor in the real application. In this study, the inspection resource and the cost are both important for stakeholders. The IARC model can generate compromise solutions to make a trade-off between the cost and the load balance. Decision-makers can choose one among the multiple solutions generated by modified NSGA-II algorithms.

The experiment results show that the modified NSGA-II algorithm has a good performance in terms of diversity and convergence compared with NSGA-II in the inspection allocation problem. The modified algorithm can obtain more solutions that perform better in an objective space by using the HV metrics. It proves the effectiveness of adaptive mutation operators, which can improve the neighborhood search capability of the modified NSGA-II.

Regarding future work, more influence factors will be included in the IARC model as the objectives, such as energy efficiency, and a multi-objective optimization model will be 
TABle 5: The result comparison of two methods by running 10 times.

\begin{tabular}{|c|c|c|c|c|c|c|c|c|c|}
\hline \multirow{2}{*}{ Instances } & \multicolumn{4}{|c|}{ Modified NSGA-II } & \multicolumn{4}{|c|}{ NSGA-II } & \multirow{2}{*}{ Difference } \\
\hline & Mean & var & $N$ & Time & Mean & var & $N$ & Time & \\
\hline I1_A & 0.56 & $3.1 E-02$ & 4 & 5.5 & 0.59 & $1.0 E-02$ & 3.1 & 5.1 & $-5.40 \%$ \\
\hline I1_B & 0.73 & $5.0 E-03$ & 5 & 13.3 & 0.73 & $6.4 E-03$ & 3.4 & 12.3 & $-0.59 \%$ \\
\hline I2_A & 0.89 & $8.2 E-04$ & 5 & 10.3 & 0.89 & $7.0 E-04$ & 4.3 & 9.5 & $0.04 \%$ \\
\hline I2_B & 0.99 & $1.4 E-05$ & 4 & 24.1 & 0.98 & $4.7 E-05$ & 2.6 & 20.5 & $0.74 \%$ \\
\hline I2_AB & 0.98 & $6.7 E-05$ & 8 & 20.3 & 0.95 & $4.0 E-04$ & 4.6 & 18.7 & $2.84 \%$ \\
\hline I4_A & 0.88 & $2.4 E-03$ & 7 & 23.1 & 0.85 & $3.8 E-03$ & 2.8 & 21.4 & $3.66 \%$ \\
\hline I4_B & 0.99 & $4.2 E-05$ & 9 & 42.9 & 0.94 & $1.4 E-04$ & 4.4 & 39.5 & $5.05 \%$ \\
\hline I4_AB & 0.98 & $5.2 E-05$ & 8 & 35.2 & 0.93 & $2.0 E-03$ & 4.4 & 32.3 & $4.83 \%$ \\
\hline I6_A & 0.98 & $2.4 E-04$ & 5 & 25.7 & 0.96 & $5.4 E-04$ & 3.6 & 24.0 & $1.79 \%$ \\
\hline I6_B & 0.98 & $1.4 E-04$ & 7 & 56.4 & 0.95 & $2.5 E-04$ & 5.4 & 49.6 & $3.57 \%$ \\
\hline I6_AB & 0.99 & $1.0 E-04$ & 9 & 45.6 & 0.95 & $2.4 E-04$ & 4.3 & 43.8 & $3.97 \%$ \\
\hline I8_A & 0.99 & $4.8 E-06$ & 5 & 32.0 & 0.97 & $3.7 E-04$ & 3.7 & 28.4 & $2.59 \%$ \\
\hline I8_B & 0.95 & $4.6 E-04$ & 9 & 90.1 & 0.88 & $8.1 E-04$ & 6.7 & 82.7 & $7.27 \%$ \\
\hline I8_AB & 0.97 & $1.7 E-04$ & 9 & 66.2 & 0.92 & $1.1 E-03$ & 5.9 & 63.3 & $5.27 \%$ \\
\hline I10_A & 0.98 & $2.1 E-04$ & 6 & 61.3 & 0.91 & $4.3 E-03$ & 3.8 & 58.7 & $7.65 \%$ \\
\hline I10_B & 0.96 & $9.4 E-04$ & 7 & 132.5 & 0.91 & $5.2 E-04$ & 6.4 & 117.3 & $6.06 \%$ \\
\hline I10_AB & 0.95 & $1.1 E-03$ & 7 & 74.5 & 0.88 & $3.1 E-03$ & 6.8 & 70.8 & $7.73 \%$ \\
\hline
\end{tabular}

Note. mean $=$ the mean value of $\mathrm{HV}$ of 10 runs; var = the variance of $\mathrm{HV}$ of 10 runs. $N=$ the average number of solutions in nondominated solutions set.

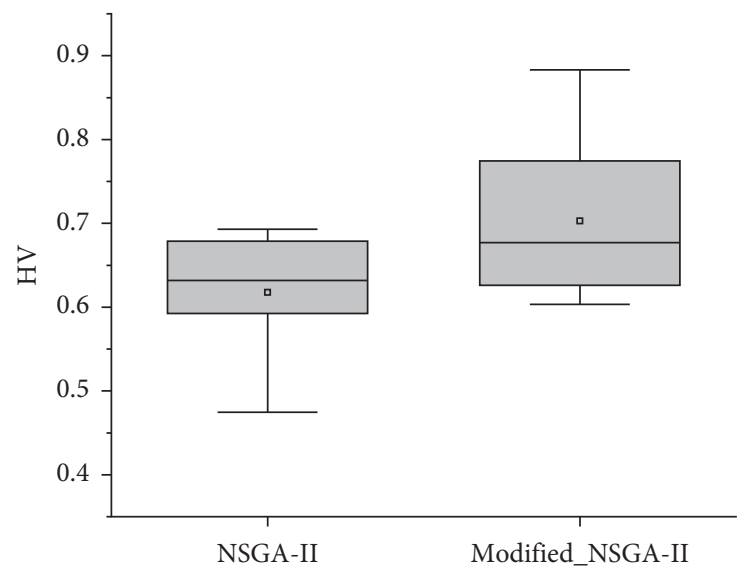

Figure 5: Box plot of HV metric in example I4_A.

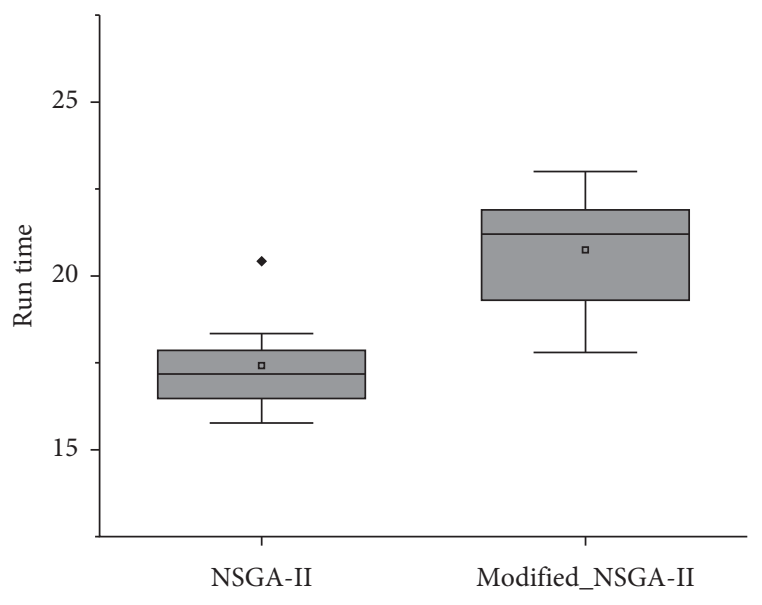

FIgURE 6: Box plot of runtime in example I4_A.

built to find the optimum inspection solutions. Besides, a production system normally has many activities, such as production, inspection, and maintenance, and these
TABLE 6: The average changes of weight value for mutation operators.

\begin{tabular}{lccc}
\hline Mutation methods & RIC & RER & MLR \\
\hline Final weight value & 10.1 & 4.1 & 22.1 \\
\hline
\end{tabular}

activities need to be scheduled to improve the efficiency of the whole system. Thus, joint scheduling of production, inspection, and maintenance activities need further research. In terms of algorithm, the proposed modified algorithm could be employed in similar problems, and it is interesting to investigate the performance in other MOP problems.

The experiment result shows that the proposed IARC model has a certain guidance meaning for the realization of the intelligent, automation inspection planning systems. Although the model still has a long way to go from the practical application as it has some hypotheses, it is an interesting direction that integrates the IARC model into the IPS, which is commercially used in factories. By combining the inspection resources in the industrial field, the model can 
provide real-time inspection planning solutions, which can support the decision-making process.

\section{Conclusions}

This paper researched inspection allocation problems considering inspection resources. The main contributions are as follows:

(1) Based on the characteristics of the FMS, an IARC model is proposed to analyse the possibility and probability value of the product in the product flow by using the inspection capability model. The IARC mixed-integer programming model is built to tradeoff the total cost, the load of the inspection station.

(2) To solve the multi-objective optimization model, the NSGA-II algorithm is improved. A four-layer coding scheme, the improved elite strategy, and mutation operators are integrated into NSGA-II. The neighborhood research capability of the methods is enhanced so that the solutions do not fall into the first layer of the Pareto solution prematurely. Finally, the simulation experiment was conducted. The result verified the effectiveness of the IARC model, the performance of the modified NSGA-II in terms of diversity and convergence.

\section{Data Availability}

The data used to support the findings of this study are available from the corresponding author upon reasonable request.

\section{Conflicts of Interest}

The authors declare no conflicts of interest.

\section{Acknowledgments}

This research was supported by New Intelligent Manufacturing Model Research Program of the Ministry of Industry and Information Technology (Grant No. NISM20180123), the National Civil Aircraft Digital Manufacturing Project (Grant No. GXMJ201503A009), and Beijing digital manufacturing key laboratory project (Grant No. BDMLBH2016002).

\section{References}

[1] M. Mohammadi, "Mathematical modelling of a robust inspection process plan: Taguchi and Monte Carlo methods," International Journal of Production Research, vol. 53, no. 7, pp. 2202-2224, 2015.

[2] W. Winchell, Inspection and Measurement in Manufacturing: Keys to Process Planning and Improvement, Society of Manufacturing Engineers, Southfield, MI, USA, 1996.

[3] Y.-R. Shiau, "Quick decision-making support for inspection allocation planning with rapidly changing customer requirements," The International Journal of Advanced Manufacturing Technology, vol. 22, no. 9, pp. 633-640, 2003.
[4] L. Solano, F. Romero, and P. Rosado, "An ontology for integrated machining and inspection process planning focusing on resource capabilities," International Journal of Computer Integrated Manufacturing, vol. 29, no. 1, pp. 1-15, 2016.

[5] G. F. Lindsay and A. B. Bishop, "Allocation of screening inspection effort-a dynamic-programming approach," Management Science, vol. 10, no. 2, pp. 342-352, 1964.

[6] E. G. Hurst, "Imperfect inspection in a multistage production process," Management Science, vol. 20, no. 3, pp. 378-384, 1973.

[7] Y.-R. Shiau, M.-H. Lin, and W.-C. Chuang, "Concurrent process/inspection planning for a customized manufacturing system based on genetic algorithm," The International Journal of Advanced Manufacturing Technology, vol. 33, no. 7-8, pp. 746-755, 2007.

[8] P. Korytkowski, "A genetic algorithm with tournament selection for optimising inspection allocation in multiproduct multistage production systems," International Journal of Simulation and Process Modelling, vol. 6, no. 3, pp. 238-244, 2011.

[9] Y.-R. Shiau, "Inspection allocation planning for a multiple quality characteristic advanced manufacturing system," The International Journal of Advanced Manufacturing Technology, vol. 21, no. 7, pp. 494-500, 2003.

[10] Y. Narahari and L. Khan, "Modeling reentrant manufacturing systems with inspection stations," Journal of Manufacturing Systems, vol. 15, no. 6, pp. 367-378, 1996.

[11] J. Lee and S. Unnikrishnan, "Planning quality inspection operations in multistage manufacturing systems with inspection errors," International Journal of Production Research, vol. 36, no. 1, pp. 141-156, 1998.

[12] H. Rau and K.-H. Cho, "Genetic algorithm modeling for the inspection allocation in reentrant production systems," Expert Systems with Applications, vol. 36, no. 8, pp. 11287-11295, 2009.

[13] J. Faigl and L. Přeučil, "Inspection planning in the polygonal domain by Self-Organizing Map," Applied Soft Computing, vol. 11, no. 8, pp. 5028-5041, 2011.

[14] M. Mohammadi, A. Saidat, and R. Tavakkoli-Moghaddam, "A bi-objective robust inspection planning model in a multistage serial production system," International Journal of Production Research, vol. 56, no. 4, pp. 1432-1457, 2018.

[15] S. V. Volsem, "Joint optimisation of all inspection parameters for multistage processes: evolutionary algorithm and simulation," International Journal of Innovative Computing and Applications, vol. 2, no. 3, pp. 170-177, 2010.

[16] Y. Feng, "Monarch butterfly optimization: a comprehensive review," Expert Systems with Applications, vol. 168, Article ID 114418, 2020.

[17] J.-H. Yi, "An improved NSGA-III algorithm with adaptive mutation operator for Big Data optimization problems," Future Generation Computer Systems, vol. 88, pp. 571-585, 2018.

[18] Y. Zhang, "Enhancing MOEA/D with information feedback models for large-scale many-objective optimization," Information Sciences, vol. 522, pp. 1-16, 2020.

[19] W. Deng, "Differential evolution algorithm with wavelet basis function and optimal mutation strategy for complex optimization problem," Applied Soft Computing, vol. 100, Article ID 106724, 2021.

[20] W. Deng, "An enhanced MSIQDE algorithm with novel multiple strategies for global optimization problems," IEEE Transactions on Systems, Man, and Cybernetics: Systems, 2020.

[21] A. Verduzco, J. R. Villalobos, and B. Vega, "Informationbased inspection allocation for real-time inspection systems," 
Journal of Manufacturing Systems, vol. 20, no. 1, pp. 13-22, 2001.

[22] S. Van Volsem, W. Dullaert, and H. Van Landeghem, "An evolutionary algorithm and discrete event simulation for optimizing inspection strategies for multi-stage processes," European Journal of Operational Research, vol. 179, no. 3, pp. 621-633, 2007.

[23] G. Galante and G. Passannanti, "Integrated approach to part scheduling and inspection policies for a job shop manufacturing system," International Journal of Production Research, vol. 45, no. 22, pp. 5177-5198, 2007.

[24] P. Korytkowski, B. Malachowski, and T. Wisniewski, "Genetic algorithm for optimization of inspection stations allocation in multi-product manufacturing systems," in Proceedings of the 2009 International Conference on Computers \& Industrial Engineering, IEEE, Troyes, France, 2009.

[25] Y.-R. Shiau, "Inspection resource assignment in a multistage manufacturing system with an inspection error model," International Journal of Production Research, vol. 40, no. 8, pp. 1787-1806, 2002.

[26] K. Deb, "A fast and elitist multiobjective genetic algorithm: NSGA-II," IEEE Transactions on Evolutionary Computation, vol. 6, no. 2, pp. 182-197, 2002.

[27] F. Sahling, "Solving a multi-level capacitated lot sizing problem with multi-period setup carry-over via a fix-andoptimize heuristic," Computers \& Operations Research, vol. 36, no. 9, pp. 2546-2553, 2009.

[28] E. Zitzler, K. Deb, and L. Thiele, "Comparison of multiobjective evolutionary algorithms: empirical results," Evolutionary Computation, vol. 8, no. 2, pp. 173-195, 2000.

[29] D. A. Van Veldhuizen and G. B. Lamont, "On measuring multiobjective evolutionary algorithm performance," in Proceedings of the 2000 Congress on Evolutionary Computation. CECO0 (Cat. No. 00TH8512), IEEE, La Jolla, CA, USA, 2000.

[30] G. C. Ciro, "A NSGA-II and NSGA-III comparison for solving an open shop scheduling problem with resource constraints," IFAC-PapersOnLine, vol. 49, no. 12, pp. 1272-1277, 2016.

[31] N. Beume, B. Naujoks, and M. Emmerich, "SMS-EMOA: multiobjective selection based on dominated hypervolume," European Journal of Operational Research, vol. 181, no. 3, pp. 1653-1669, 2007.

[32] X. Zou, "new evolutionary algorithm for solving many-objective optimization problems," IEEE Transactions on Systems, Man, and Cybernetics, Part B (Cybernetics), vol. 38, no. 5, pp. 1402-1412, 2008.

[33] Q. Zhang and H. Li, "MOEA/D: a multiobjective evolutionary algorithm based on decomposition," IEEE Transactions on Evolutionary Computation, vol. 11, no. 6, pp. 712-731, 2007. 